Research Article

\title{
Heat Transfer in Boundary Layer Magneto-Micropolar Fluids with Temperature-Dependent Material Properties over a Stretching Sheet
}

\author{
Ephesus O. Fatunmbi $\mathbb{D}^{1}$ and Samuel S. Okoya ${ }^{2}$ \\ ${ }^{1}$ Department of Mathematics and Statistics, Federal Polytechnic, Ilaro, Nigeria \\ ${ }^{2}$ Department of Mathematics, Obafemi Awolowo University, Ile-Ife, Nigeria \\ Correspondence should be addressed to Ephesus O. Fatunmbi; olusojiephesus@yahoo.com
}

Received 23 December 2019; Revised 31 March 2020; Accepted 15 April 2020; Published 5 May 2020

Academic Editor: Veronica Calado

Copyright () 2020 Ephesus O. Fatunmbi and Samuel S. Okoya. This is an open access article distributed under the Creative Commons Attribution License, which permits unrestricted use, distribution, and reproduction in any medium, provided the original work is properly cited.

\begin{abstract}
The process of heat transfer in boundary layer magneto-micropolar fluid with temperature-dependent material properties past a flat stretching sheet in a porous medium is investigated in this study. Two distinct cases of boundary heating conditions are analyzed for the heat transfer in this work, viz., prescribed surface temperature (PST) and prescribed heat flux (PHF). With the aid of similarity conversion analysis, the formulated equations of the flow and heat transfer have been translated into a system of nonlinear ordinary differential equations. Subsequently, Runge-Kutta-Fehlberg integration scheme in company of shooting techniques employed to obtain numerical solutions to the reduced equations. The findings are graphically illustrated and discussed in view of the two cases of boundary heating, while the results for the physical quantities of engineering concern are tabulated for various controlling parameters. In the limiting situations, the results generated are compared favourably with the earlier reported data in the literature, while the numerical solutions demonstrate a reduction in the rate of heat transfer $\left(N u_{x}^{\star}\right)$ and the viscous drag $\left(C_{f}^{\star}\right)$ for both PST and PHF conditions with growth in the magnitude of material parameter $K$.
\end{abstract}

\section{Introduction}

The dynamics of non-Newtonian fluids has gained preeminence in the recent times owing to their potential applications both in engineering and industrial operations. For instance, in crude oil extraction, food processing, extrusion of polymers, and syrup drugs. The characteristics feature of non-Newtonian fluids might differ from that of Newtonian fluids in diverse ways: the shear thinning or thickening of the fluid, the tendency to yield stress and display stress relaxation, and to creep. Most often, the viscosity depends on the shear rate or shear rate history. Such fluids include suspension solutions, biological fluids (e.g., saliva and blood), lubricants, polymer solutions and colloids, and emulsions [1].

The diversity in the nature of non-Newtonian fluids gives room for the existence of different models, each of them capturing particular characteristics of the fluid since no single model can completely capture all the features of nonNewtonian fluids. Prominent among the non-Newtonian fluid models include the micropolar fluid, Maxwell fluid, Walters-B fluid, Casson fluid, Eyring-Powell fluid, and Jeffery fluid [1]. Eringen [2,3] initiated the concept of micropolar fluid which is a subclass of simple microfluids. This concept explains fluids with microstructures. In the physical description, these fluids are made up of rigid, barlike, or spherical particles suspended in a viscous medium. Examples are polymeric fluids, animal blood, exotic lubricants, body fluids, colloids, and liquid crystals. This concept is interesting and attractive as it represents a generalization of the classical Navier-Stokes concept alongside its practical applications in different areas of science, engineering, and technology, for instance, in biomedical fluid engineering, synovial lubrication, drug suspension in pharmacology, 
arterial blood flow, and extrusion of polymer fluids [4]. More so, Ahmadi [5] as well as Hayat et al. [6] pointed out that this class of fluids offers a mathematical framework for analyzing many complex and complicated fluids including polymeric fluids, fluid suspensions, exotic lubricants, and paints. A thorough review on the theory and applications of the micropolar fluid was presented by Lukaszewicz [7], while the boundary layer flows of such fluids were first reported by Peddieson and McNitt and Wilson $[8,9]$.

Studies involving fluid flow analysis with heat transfer characteristics activated by a stretching sheet have consequential engineering and industrial applications which include wire drawing, extrusion of polymer sheet, glass blowing, and textile and paper production. Pioneering such study, Crane [10] reported a closed form analytical solution where the velocity is proportional to the distance from the fixed origin. Thereafter, various researchers have extended such study under different situations. Gupta and Gupta [11] considered the impact of suction or blowing on the heat and mass transfer due to a stretching sheet being influenced by constant surface temperature. Furthermore, Chakrabarti and Gupta [12] offered a similarity solution to such problem with the impact of a transverse magnetic field with constant suction and temperature. Grubka and Bobba [13] derived closed form solutions of variable temperature on the transfer of heat prompted by a stretching sheet with a prescribed surface temperature, while Elbashbeshy and Bazid [14] engaged numerical tool via Runge-Kutta integration technique cum shooting technique to address such problem in a porous medium. In addition, Eldabe et al. [15] analyzed MHD non-Newtonian micropolar fluid with heat transfer characteristics, while Fatunmbi and Odesola [16] reported such study with uniform heat flux.

The aforementioned research studies were, however, conducted with a notion that the fluid thermophysical properties are constant whereas these properties, particularly the fluid viscosity and thermal conductivity, have been found to vary with changes in temperature. The internal heat generation due to friction as well as an increase in temperature may influence these properties. In particular, the increase in temperature from $10^{\circ} \mathrm{C}$ to $50^{\circ} \mathrm{C}$ causes the viscosity of water to decrease by about $240 \%$, while the viscosity of air is $0.6924 \times 10^{-5}$ at $1000 \mathrm{~K}, 1.3289$ at $2000 \mathrm{~K}, 2.286$ at $4000 \mathrm{~K}$, and 3.625 at $8000 \mathrm{~K}$ [17]. Hence, the assumption of constant fluid properties may not yield accurate results as remarked by Postelnicu et al. [18]. For accurate prediction, therefore, it is essential to find out the possible effects of temperature-dependent thermophysical properties of the fluid on heat transfer processes. The application of such study includes hot rolling, paper and textile production, process of wire drawing, and drawing of plastic films. To this end, various researchers [19-21] have investigated the influence of variable fluid properties along stretching surfaces for different fluids employing diverse heating conditions and geometry.

Fluid flow as well as heat transfer in porous materials has consequential applications in diverse industrial and engineering works ranging from geothermal energy extractions, petroleum technology, ceramic and ground water hydrology, and solar heating systems to polymer technology and nuclear reactor. Due to these crucial applications, Ahmed and Rashed [22] as well as Amanulla et al. [23] recently reported such cases. These effects have also been analyzed by various researchers [24-26].

The understanding of the impact of radiation in heat transfer processes is quite essential for the construction and development of appropriate equipment in various energy conversion and engineering operations such as in gas turbines and nuclear power plants, as well as in diverse propulsion devices for aircraft [27]. This effect has been reported by a number of researchers [28-30]. Besides, the nature of heat transfer is also dictated by the type of wall heating condition that is applied in industrial and engineering processes. In the light of this, Gholinia et al. [31] applied PST to examine the movement of nanofluid through a circular cylinder with magnetic field impact. Cortell [30] engaged PST and PHF heating conditions to investigate heat transfer activated by the nonlinear stretching sheet in the presence of thermal radiation, suction/injection, and viscous dissipation. Similarly, Gireesha et al. [32] applied both heating conditions to explore the nature of heat transfer in a dusty fluid, while numerical investigation of the influence of variable fluid properties on such problem was carried out by Pal and Mondal [33].

Nevertheless, these studies have only been conducted with Newtonian fluids without due consideration for the non-Newtonian fluids especially the micropolar fluid inspite of its immense applications in engineering, science, and technology as highlighted earlier. Thus, it becomes imperative to examine the boundary layer heat transfer processes on such fluid with relevant application in polymer industries. In particular, this study focuses on numerical analysis of boundary layer heat transfer characteristics in MHD nonNewtonian micropolar fluid being influenced by temperature-dependent properties, viscous dissipation, and thermal radiation under the application of both PST and PHF wall heating conditions. The organization of the study is as follows: Section 1 is the introduction to the study, while Section 2 narrates the mathematical formulation of the work, Section 3 portrays the method of solution to the transformed governing equations, Section 4 covers the discussion of results, while the conclusion is explained in Section 5.

\section{Mathematical Formulation}

The physical model in this work is portrayed in Figure 1 showing a two-dimensional, steady, and incompressible flow of micropolar fluid passing a stretching sheet in a porous medium being influenced by thermal radiation, internal heat generation, or absorption, as well as viscous dissipation. Here, the flow direction is towards $\bar{x}$ axis, whereas $\bar{y}$ axis is normal to it with $\bar{u}$ and $\bar{v}$ designating components of velocity in the direction of $\bar{x}$ and $\bar{y}$. Meanwhile, a constant magnetic field $\mathbf{B}=\left(0, B_{0}, 0\right)$ parallel to $\bar{y}$ axis is imposed perpendicular to flow direction, whereas the induced magnetic field is negligible. At the sheet, the velocity is taken to be $\bar{u}_{w}=a \bar{x}$, while the external 


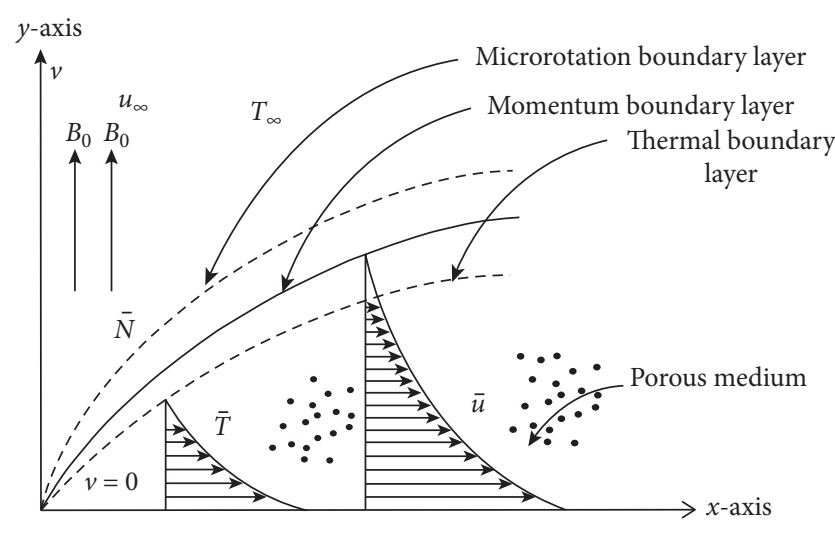

$u_{w}=a \bar{x}, \bar{T}=T_{w}=T_{\infty}+A(\bar{x} / L)^{2},-K_{\infty}(\partial \bar{T} / \partial \bar{y})=q_{w}=D(\bar{x} / L)^{2} \longrightarrow$

Figure 1: Flow geometry.

flow velocity is taken to be zero. More so, the prescribed surface temperature (PST) $\bar{T}=T_{w}=T_{\infty}+A(\bar{x} / L)^{2}$ and the prescribed heat flux (PHF) $-k_{\infty}(\partial \bar{T} / \partial \bar{y})=q_{w}=D(\bar{x} / L)^{2}$ are assumed as the boundary heating conditions. Also, the angular velocity of the microparticles $\mathbf{N}=(0,0, \bar{N}(\bar{x}, \bar{y}))$ is applied, while both viscosity and thermal conductivity are temperature-dependent.

In the light of the aforementioned assumptions and boundary layer approximation, the governing equations are $[19,20]$

$$
\begin{aligned}
\frac{\partial \bar{u}}{\partial \bar{x}}+\frac{\partial \bar{v}}{\partial \bar{y}}= & 0 \\
\bar{u} \frac{\partial \bar{u}}{\partial \bar{x}}+\bar{v} \frac{\partial \bar{u}}{\partial \bar{y}}= & \frac{1}{\rho} \frac{\partial}{\partial \bar{y}}\left(\mu \frac{\partial \bar{u}}{\partial \bar{y}}\right)+\frac{\kappa}{\rho} \frac{\partial^{2} \bar{u}}{\partial \bar{y}^{2}}+\frac{\kappa}{\rho} \frac{\partial \bar{N}}{\partial \bar{y}} \\
& -\frac{(\mu+\kappa)}{\rho K_{p}} \bar{u}-\frac{\sigma B_{o}^{2}}{\rho} \bar{u} \\
\bar{u} \frac{\partial \bar{N}}{\partial \bar{x}}+\bar{v} \frac{\partial \bar{N}}{\partial \bar{y}}= & \frac{\gamma}{\rho j} \frac{\partial^{2} \bar{N}}{\partial \bar{y}^{2}}-\frac{\kappa}{\rho j}\left(2 \bar{N}+\frac{\partial \bar{u}}{\partial \bar{y}}\right) \\
\bar{u} \frac{\partial \bar{T}}{\partial \bar{x}}+\bar{v} \frac{\partial \bar{T}}{\partial \bar{y}}= & \frac{1}{\rho C_{p}} \frac{\partial}{\partial \bar{y}}\left(k \frac{\partial \bar{T}}{\partial \bar{y}}\right)-\frac{1}{\rho C_{p}} \frac{\partial q_{r}}{\partial \bar{y}}+\frac{(\mu+\kappa)}{\rho C_{p}}\left(\frac{\partial \bar{u}}{\partial \bar{y}}\right)^{2} \\
& +\frac{Q^{\star}}{\rho c_{p}}\left(\bar{T}-T_{\infty}\right) .
\end{aligned}
$$

In line with several authors [34-36], the temperaturedependent viscosity $\mu$ is expressed as

$$
\mu(\bar{T})=\frac{\mu_{0}}{\left[1+\beta\left(\bar{T}-T_{\infty}\right)\right]} .
$$

Also, the variation of the thermal conductivity $k$ with temperature can be expressed in an approximately linear form as $[33,37,38]$

$$
k(\bar{T})\left(T_{w}-T_{\infty}\right)=k_{\infty}\left[\left(T_{w}-T_{\infty}\right)+\epsilon\left(\bar{T}-T_{\infty}\right)\right] .
$$

Similarly, various researchers $[21,39]$ have shown that the structure of radiative heat flux can be described as

$$
q_{r}=-\frac{4 \sigma^{\star}}{3 \alpha^{\star}} \frac{\partial \overline{T^{4}}}{\partial \bar{y}} .
$$

Equations (1)-(3) have the following boundary conditions:

$$
\begin{gathered}
\bar{y}=0: \bar{u}=u_{w}=a \bar{x}, \bar{v}=0, \quad \bar{N}=-n \frac{\partial \bar{u}}{\partial \bar{y}}, \\
\bar{y} \longrightarrow \infty: \bar{u} \longrightarrow 0, \quad \bar{N} \longrightarrow 0 .
\end{gathered}
$$

The conditions at the boundary for the solution of energy equation (4) are particularly determined by the nature of heating process applied. In this study, we have imposed two different cases: (i) PST and (ii) PHF. Therefore, following $[30,33]$, the boundary conditions for equation (4) are

$$
\begin{aligned}
\bar{y} & =0:\left(\text { i) } \bar{T}=T_{w}=T_{\infty}+A\left(\frac{\bar{x}}{L}\right)^{2},\right. \\
\text { (ii) } k_{\infty} \frac{\partial \bar{T}}{\partial \bar{y}} & =q_{w}=D\left(\frac{\bar{x}}{L}\right)^{2}, \\
\bar{y} & \longrightarrow \infty: \bar{T} \longrightarrow T_{\infty} .
\end{aligned}
$$

In equations (1)-(9), the fluid density is represented by $\rho$, while $\bar{u}$ and $\bar{v}$ represent the velocity components in the direction of $\bar{x}$ and $\bar{y}$, respectively. The vortex or microrotation viscosity is represented as $\kappa, \bar{N}$ defines the microrotation component, $\bar{T}$ denotes the fluid temperature, while $B_{o}$ is the magnetic field strength, and the specific heat at constant pressure is $C_{p}$. More so, $T_{w}$ and $T_{\infty}$ stand for the sheet temperature and the free stream temperature, respectively. The heat source/sink is symbolized by $Q^{\star}$, while $K_{p}$ stands for the permeability of the porous medium, and $a$ is a constant, $a>0$.

More so, $j$ represents the microinertia density, while the spin gradient viscosity is described as $\gamma$ which has been defined by various authors as $\gamma=(\mu+\kappa / 2) j$ (see Ahmadi [5] and Ishak [29]); also, $\sigma^{\star}$ and $\alpha^{\star}$ define the Stefan-Boltzmann constant and the mean absorption coefficient, respectively.

Similarly, $n$ in equation (5) defines the micropolar boundary parameter with $0 \leq n \leq 1$ [29]. Here, $n=0$, it implies that $\bar{N}=0$, and this relates to a strong concentration of the microelements at the solid boundary, while $n=1 / 2$ designates the case of weak concentration (see Peddieson [40] and Jena and Mathur [41]). Furthermore, Ahmadi [5] suggested that $n=1$ is applicable for turbulence situations in the boundary layer. In equations (5), (6), and (9), $\mu_{0}$ stands for viscosity at reference temperature, $k_{\infty}$ represents the free stream thermal conductivity, $\epsilon$ denotes the variable thermal conductivity parameter, $L$ denotes the characteristic length, $q_{w}$ defines the heat flux, while $A$ and $D$ are constants. The underlisted similarity and nondimensional variables are also introduced into governing equations (1)-(4): 


$$
\begin{aligned}
\bar{u} & =\frac{\partial \psi}{\partial \bar{y}}=a \bar{x} f^{\prime}(\eta), \\
\bar{v} & =-\frac{\partial \psi}{\partial \bar{x}}=-(a v)^{1 / 2} f(\eta), \\
\eta & =\left(\frac{a}{v}\right)^{1 / 2} \bar{y} \\
\bar{N} & =a \bar{x} h(\eta)\left(\frac{a}{v}\right)^{1 / 2}, \\
\theta & =\frac{\bar{T}-T_{\infty}}{T_{w}-T_{\infty}}, \\
T_{w}-T_{\infty} & =\left(\frac{\nu}{a}\right)^{1 / 2} \frac{q_{w}}{k_{\infty}} .
\end{aligned}
$$

With the substitution of equation (10) in equations (1)-(4) and taking cognizance of equations (5)-(7), equation (1) is valid, while equations (2)-(4) translate to

$$
\begin{gathered}
\left((1+h \theta)+(1+h \theta)^{2} K\right) f^{\prime \prime \prime}-h \theta^{\prime} f^{\prime \prime}+(1+h \theta)^{2}\left(f f^{\prime \prime}-f^{\prime 2}\right. \\
\left.+K g^{\prime}\right)-(1+h \theta)[D a+(K+M)(1+h \theta)] f^{\prime}=0 \\
\left(1+\frac{K}{2}\right) g^{\prime \prime}+f g^{\prime}-f^{\prime} g-K\left(2 g+f^{\prime \prime}\right)=0 \\
(1+h \theta)(1+\epsilon \theta+N r) \theta^{\prime \prime}+(1+h \theta) \epsilon \theta^{\prime 2}+\operatorname{PrEc} \\
\cdot(1+K(1+h \theta)) f^{\prime \prime 2}+\operatorname{Pr}\left(f \theta \prime-2 \theta f^{\prime}+Q \theta\right)(1+h \theta)=0
\end{gathered}
$$

Boundary conditions (8) similarly translate to

$$
\begin{gathered}
f^{\prime}(0)=1, \\
f(0)=0, \\
g(0)=-n f^{\prime \prime}, \\
f^{\prime}(\infty) \longrightarrow 0, \\
g(\infty) \longrightarrow 0,
\end{gathered}
$$

while boundary conditions (9) transform to

$$
\begin{aligned}
\text { (i) } \theta(0) & =1, \\
\text { (ii) } \theta^{\prime}(0) & =-1, \\
\theta(\infty) & \longrightarrow 0 .
\end{aligned}
$$

Various symbols involved in equations (11)-(13) are described as follows:

$$
\begin{aligned}
h & =\beta\left(T_{w}-T_{\infty}\right), \\
K & =\frac{\kappa}{\mu_{0}}, \\
D a & =\frac{\nu}{a K_{p}}, \\
\operatorname{Pr} & =\frac{\mu_{0} C_{p}}{k_{\infty}}, \\
M & =\frac{\sigma B_{0}^{2}}{a \rho}, \\
N r & =\frac{16 \sigma^{\star} T_{\infty}^{3}}{3 \alpha^{\star} k_{\infty}} \\
Q & =\frac{Q^{\star}}{a \rho C p}, \\
E c & =\frac{a^{2}}{C p D L^{-2}}\left(\frac{a}{v}\right)^{1 / 2}(\mathrm{PHF}), \\
E c & =\frac{a^{2}}{C p A L^{-2}}(\mathrm{PST}),
\end{aligned}
$$

where $h$ denotes the temperature-dependent viscosity parameter and $\beta>0, K$ represents the material (micropolar) parameter, $D a$ is the Darcy parameter, $P r$ stands for the Prandtl parameter, $Q$ denotes the heat source/sink parameter, $E c$ stands for the Eckert number, $\mathrm{Nr}$ defines the radiation parameter, and $M$ denotes the magnetic field parameter. It is important to note also that the size of vortex or microrotation viscosity $\kappa$ allows us to measure in certain sense the deviation of the micropolar fluid model from that of the classical Newtonian fluid model. Hence, when $\kappa=0(K=0)$, equations (2) or (11) and (4) or (13) are decoupled from equation (3) or (12). Then, the present problem as well as its solution reduces to the model of classical Newtonian fluid.

The relevant quantities of engineering concern are the skin friction coefficient, the Nusselt number, and the wall couple stress coefficient which are correspondingly described in the following equation:

$$
\begin{aligned}
C_{f} & =\frac{\tau_{w}}{\rho u_{w}^{2}}, \\
N u_{x} & =\frac{\bar{x} q_{w}}{k_{\infty}\left(T_{w}-T_{\infty}\right)}, \\
C_{s} & =\frac{\bar{x} M_{w}}{\mu j a},
\end{aligned}
$$

where $\tau_{w}=[(\mu+\kappa)(\partial \bar{u} / \partial \bar{y})+\kappa \bar{N}]_{\bar{y}=0}$ defines the wall shear stress, $q_{w}=-k_{\infty}[(1+N r)(\partial \bar{T} / \partial \bar{y})]_{\bar{y}=0}$ describes the heat flux at the surface, while $M_{w}=[\gamma(\partial \bar{N} / \partial \bar{y})]_{\bar{y}=0}$ gives the wall couple stress. 
The respective nondimensional form of the skin friction and the couple stress coefficients are (see Rahman et al. [42])

$$
\begin{aligned}
& C_{f}^{\star}=f^{\prime \prime}(0), \\
& C_{s}^{\star}=g^{\prime}(0),
\end{aligned}
$$

while the nondimensional Nusselt number for the PST and $\mathrm{PHF}$ conditions is, respectively, given as

$$
N u_{x}^{\star}=-\theta^{\prime}(0), \quad \frac{1}{\theta(0)},
$$

where

$$
\begin{aligned}
C_{f}^{\star} & =\frac{(1+h)\left(R e_{x}\right)^{(1 / 2)}}{(1+K / 2)} C_{f}, \\
N u_{x}^{\star} & =\frac{N u_{x}}{(1+N r)\left(R e_{x}\right)^{(1 / 2)}}, \\
C_{s}^{\star} & =\frac{C_{s}}{(1+K / 2)\left(R e_{x}\right)^{(1 / 2)}} .
\end{aligned}
$$

We compare our model with existing ones in the literature as depicted in Table 1.

\section{Method of Solution}

The numerical solutions of equations (11)-(13) with the associated boundary conditions (14) and (15) have been sought via shooting procedure cum Runge-Kutta-Fehlberg fourthfifth integration technique. Using this technique, a suitable finite value of $\eta \longrightarrow \infty$ has been selected, say $\eta_{\infty}$. The nonlinear set of equations (11)-(13) with order three in $f$ and order two in both $g$ and $\theta$ is reduced into a set of seven first-order simultaneous linear equations after which they are transmuted into an initial value problem by means of the shooting method. This system requires seven initial conditions for the solution;
TABLE 1: Table of comparison showing authors and the special cases of our model problem.

\begin{tabular}{lccccccccc}
\hline Author & PST & PHF & $K$ & $h$ & $\epsilon$ & $D a$ & $R$ & $E c$ & $Q$ \\
\hline Seddeek and & No & Yes & No & No & Yes & No & Yes & No & No \\
Salem [43] & & & & & & & \\
Khedr et al. [44] & No & No & Yes & No & No & No & Yes & Yes & Yes \\
Eldabe et al. [15] & No & No & Yes & No & No & Yes & No & Yes & No \\
Cortell [30] & Yes & Yes & No & No & No & No & Yes & Yes & No \\
Nandeppanavar & Yes & Yes & No & No & Yes & No & No & No & Yes \\
et al. [45] & & & & & & & & & \\
Pal and & Yes & Yes & No & Yes & Yes & No & Yes & No & No \\
Mondal [33] & & & & & & & & & \\
Waqas et al. [46] & No & Yes & Yes & No & No & No & Yes & No & Yes \\
Present work & Yes & Yes & Yes & Yes & Yes & Yes & Yes & Yes & Yes \\
\hline
\end{tabular}

however, there are four initial conditions available. The unknown initial conditions $p_{1}, p_{2}$, and $p_{3}$ are guessed to get $f^{\prime \prime}(0), g^{\prime}(0)$, and $\theta \prime(0)$ in a manner that satisfies the given conditions at the boundary. This trial procedure is applied in a bit to obtain the unknown initial conditions. As the values of $p_{1}, p_{2}$, and $p_{3}$ are obtained, the integration is carried out with those values, and the accuracy of the unknown initial conditions is cross-checked by taking a comparison between the calculated value with the given end point. This procedure is reworked again with a larger value of $\eta_{\infty}$ and continues till we obtain the solution for which successive values of the unknown initial conditions $f^{\prime \prime}(0), g^{\prime}(0)$, and $\theta \prime(0)$ produce a difference after a preferred significant digit. The final value of $\eta_{\infty}$ is then taken as suitable value of the limit $\eta \longrightarrow \infty$ for a particular set of governing parameters. More details on the shooting technique can be seen in Attili and Syam [47] as well as Mahanthesh et al. [48]. The conversions of the higher-order equations into first-order differential equations are carried out as follows.

Let

$$
\begin{aligned}
& f_{1}=f, \\
& f_{2}=f^{\prime}, \\
& f_{3}=f^{\prime \prime}, \\
& f_{4}=g \\
& f_{5}=g^{\prime}, \\
& f_{6}=\theta, \\
& f_{7}=\theta \prime \\
& f_{3}^{\prime}=\frac{h f_{7} f_{3}-f_{1} f_{3}-\left(1+h f_{6}\right)^{2}\left(f_{1} f_{3}-f_{2}^{2}+K f_{5}\right)+\left(1+h f_{6}\right)\left[D a+(K+M)\left(1+h f_{6}\right)\right] f_{2}}{\left(1+h f_{6}\right)\left(1+\left(1+h f_{6}\right) K\right)} \\
& f_{5}^{\prime}=\frac{f_{2} f_{4}+K\left(2 f_{4}+f_{3}\right)-f_{1} f_{5}}{(1+K / 2)}, \\
& f_{7}^{\prime}=\frac{-\left(1+h f_{6}\right) \epsilon f_{7}^{2}-\operatorname{PrEc}\left(1+K\left(1+h f_{6}\right)\right) f_{2}^{2}-\operatorname{Pr}\left(f_{1} f_{7}-2 f_{6} f_{2}+Q f_{6}\right)\left(1+h f_{6}\right)}{\left(1+\epsilon f_{6}+N r\right)\left(1+h f_{6}\right)},
\end{aligned}
$$


and the conditions at the boundary also translate to

$$
\begin{aligned}
f_{1}(0) & =0, \\
f_{2}(0) & =1, \\
f_{3}(0) & =p_{1}, \\
f_{4}(0) & =-n f_{3}(0), \\
f_{5}(0) & =p_{2}, \\
f_{6}(0) & =1, \\
f_{7}(0) & =p_{3}, \\
f_{2}(\infty) & \longrightarrow 0 \\
f_{4}(\infty) & \longrightarrow 0, \\
f_{6}(\infty) & \longrightarrow 0 .
\end{aligned}
$$

\section{Results and Discussion}

To judge the accuracy of the numerical results obtained in this work, we have compared our solutions relating to $C_{f}^{\star}$ with the data given by Kumar [49] and Tripathy et al. [50] for a coupling situation as presented in Table 2. A good relationship exists between the present results with those authors in the limiting situations. Besides, Table 3 portrays a comparison of the Nusselt number $N u_{x}^{\star}$ for variations in Prandtl number $P r$ for the PST case with the reports given previously by Chen [51] and Seddeek and Salem [43], as well as Pal and Mondal [33] in some limiting conditions. The comparisons conform well with the earlier reported works as depicted in Table 3. Furthermore, the response of each of the controlling physical parameters has also been displayed through Figures 2-9 while that of $C_{f}^{\star}, N u_{x}^{\star}$, and $C_{s}^{\star}$ has been tabulated for effective analysis and discussion.

In Table 4, the reactions of some selected controlling parameters on $C_{f}^{\star}, N u_{x}^{\star}$, and $C_{s}^{\star}$ for the two cases investigated have been presented. The parameters investigated here are the temperature-dependent viscosity $h$, material (micropolar) parameter $K$, as well as Prandtl number $\operatorname{Pr}$. Obviously, it is seen that, as the material (micropolar) parameter $K$ appreciates in magnitude, a noticeable damping trend occurs in both $C_{f}^{\star}$ and $C_{s}^{\star}$ as well as in $N u_{x}^{\star}$ for both cases examined.

From the trends in Table 4, indication emerges that the micropolar fluid term $K$ tends to reduce the viscous drag and as well lowers the heat transfer at the surface of the sheet for both cases. Furthermore, observation shows that a rise in $\operatorname{Pr}$ enhances the transfer of heat at the sheet surface for the two cases, whereas the friction on the skin and the wall couple stress coefficients for both cases reacted differently. For the PST case, both $C_{f}^{\star}$ and $C_{s}^{\star}$ increase as $\operatorname{Pr}$ rises, whereas the trend was reversed for the PHF case, where both $C_{f}^{\star}$ and $C_{s}^{\star}$ decrease. It is noticed as well that the growth in the magnitude of the viscosity parameter $h$ boost $C_{f}^{\star}$ and $C_{s}^{\star}$ for both wall heating conditions, whereas the rate of heat transfer $N u_{x}^{\star}$ declines in both situations with growth in the magnitude of $h$. These observations are in consonance with those of Das [52].

Table 5 shows the impact of some of the physical parameters on $C_{f}^{\star}, N u_{x}^{\star}$, and $C_{s}^{\star}$ for both cases. The
TABle 2: Comparison of values of $C_{f}^{\star}$ with Kumar [49] and Tripathy et al. [50] for variation in $K, M$, and $D a$ when $n=0.5$.

\begin{tabular}{cccccc}
\hline$K$ & $M$ & $D a$ & Kumar [49] & Tripathy et al. [50] & Present \\
\hline 0.0 & 0.0 & 0.0 & 1.000000 & 1.000008 & 1.0000084 \\
0.5 & 0.0 & 0.0 & 0.880200 & 0.901878 & 0.8994515 \\
0.5 & 1.0 & 0.0 & 1.209900 & 1.250358 & 1.2496132 \\
0.5 & 1.0 & 1.0 & - & 1.510062 & 1.5127320 \\
0.0 & 0.5 & 0.0 & 1.189000 & 1.225590 & 1.2257448 \\
1.0 & 0.5 & 0.0 & 0.997600 & 0.995088 & 0.9919970 \\
1.0 & 0.5 & 1.0 & - & 1.2651260 & 1.2646592 \\
\hline
\end{tabular}

TABle 3: Values of $N u_{x}^{\star}$ for variations in $\operatorname{Pr}$ as compared with published works when $M=D a=E c=K=H=B=\xi=h=$ $R=0$.

\begin{tabular}{lcccc}
\hline $\operatorname{Pr}$ & $\begin{array}{c}\text { Chen } \\
{[51]}\end{array}$ & $\begin{array}{c}\text { Seddeek and } \\
\text { Salem [43] }\end{array}$ & $\begin{array}{c}\text { Pal and } \\
\text { Mondal [33] }\end{array}$ & $\begin{array}{c}\text { Present } \\
\text { results }\end{array}$ \\
\hline 0.72 & 1.08853 & 1.08852 & 1.088548 & 1.08852691 \\
1.00 & 1.33334 & 1.33333 & 1.353545 & 1.33333333 \\
3.00 & 2.50972 & 250972 & 2.507856 & 2.50972521 \\
7.00 & 3.97150 & 3.97150 & - & 3.97151157 \\
10.00 & 4.79686 & 4.97151 & 4.795510 & 4.79687327 \\
\hline
\end{tabular}

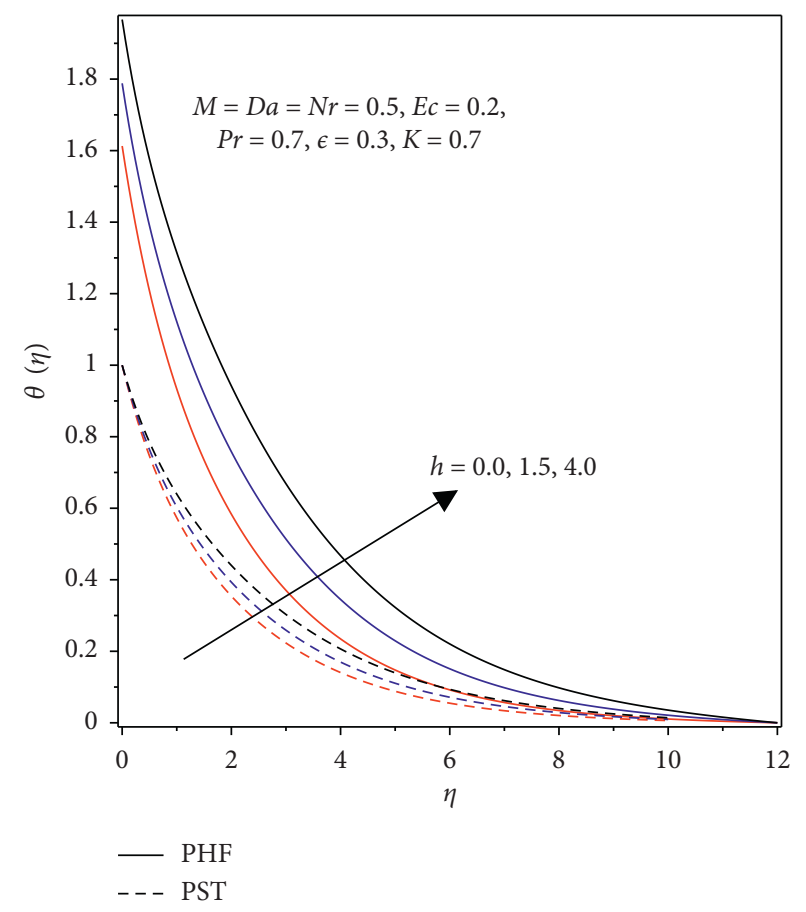

FIgURE 2: Impact of viscosity parameter $h$ on the field of temperature.

physical parameters investigated here are the temperature-dependent thermal conductivity $\epsilon$, Eckert number $E c$, and the thermal radiation parameter $N r$. Evidently, as noticed from this table, with a boost in the magnitude of $\epsilon, E \mathcal{c}$, and $N r$, a diminishing reaction occurs in $C_{f}^{\star}$ and $C_{s}^{\star}$ as well as in $N u_{x}^{\star}$ for the PST case. However, advancing the magnitude of $\epsilon, E c$, and $N r$ accelerates both $C_{f}^{\star}$ and $C_{s}^{\star}$ for the PHF case, while declining effect is noticed for that of $N u_{x}^{\star}$. 


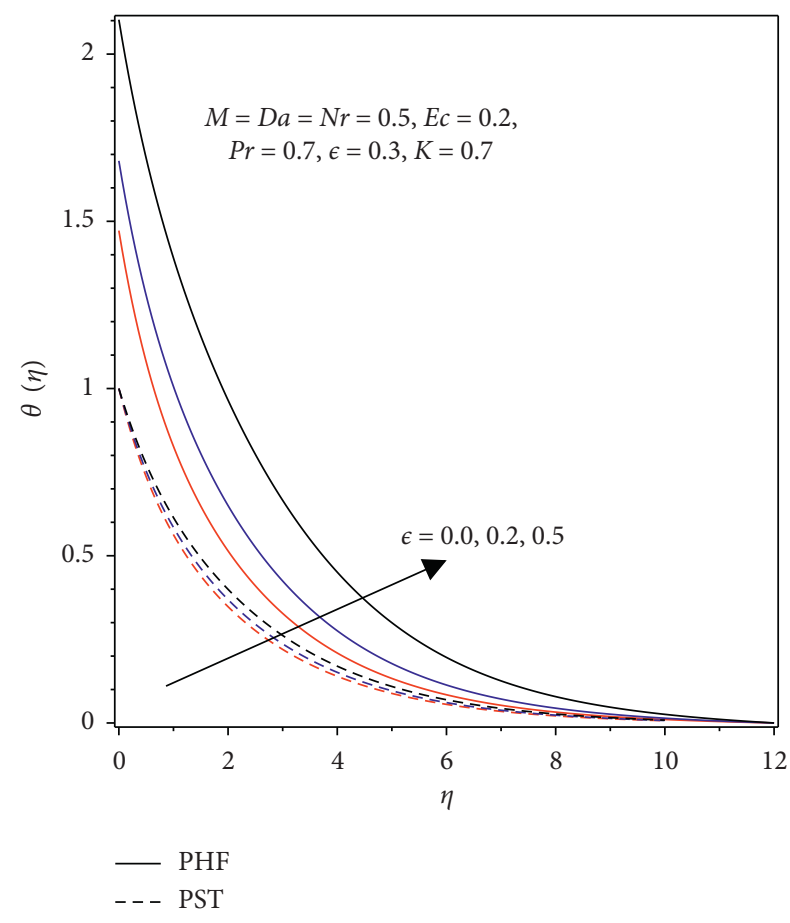

FIgURE 3: Thermal conductivity parameter $\epsilon$ variation with temperature profiles.

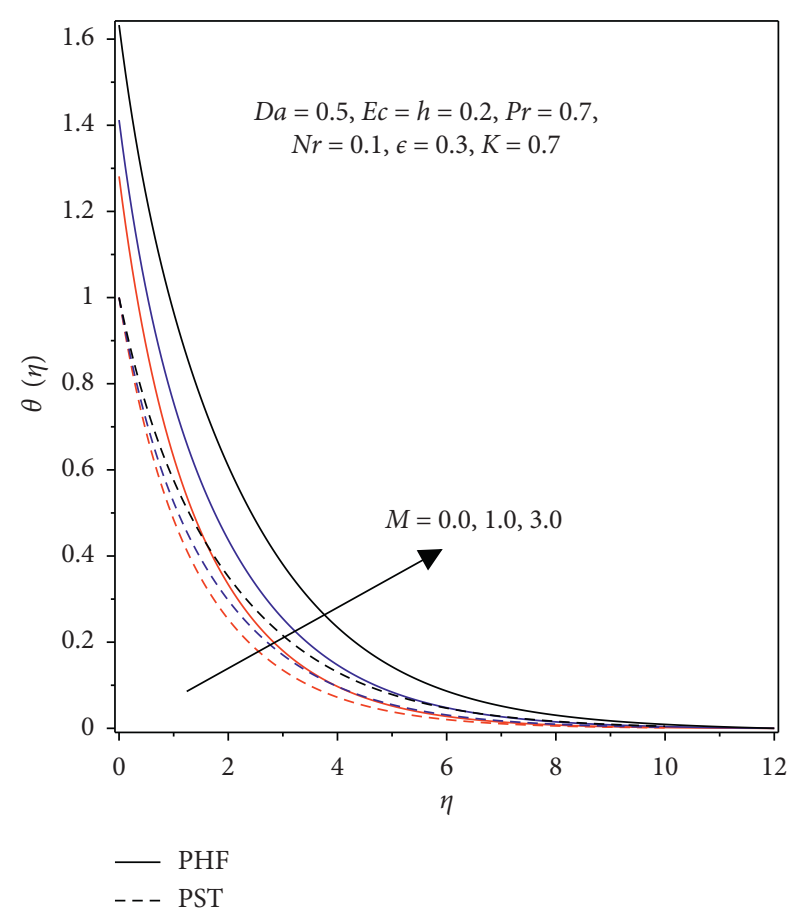

Figure 4: Magnetic field parameter $M$ variation with temperature distribution.

From the plot in Figure 2, the reaction of variable viscosity parameter $h$ evidently reveals that a growth in $h$ favours the temperature distribution and as well thickens the thermal boundary layer thickness for both cases. Higher

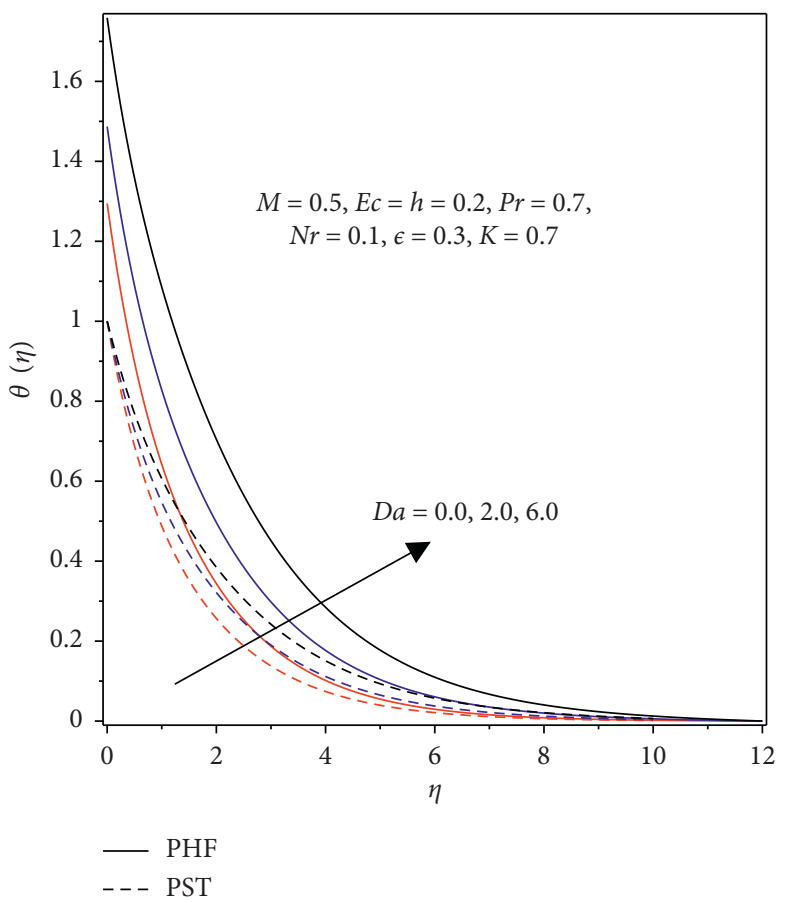

Figure 5: Variation of Darcy term $\mathrm{Da}$ on temperature field.

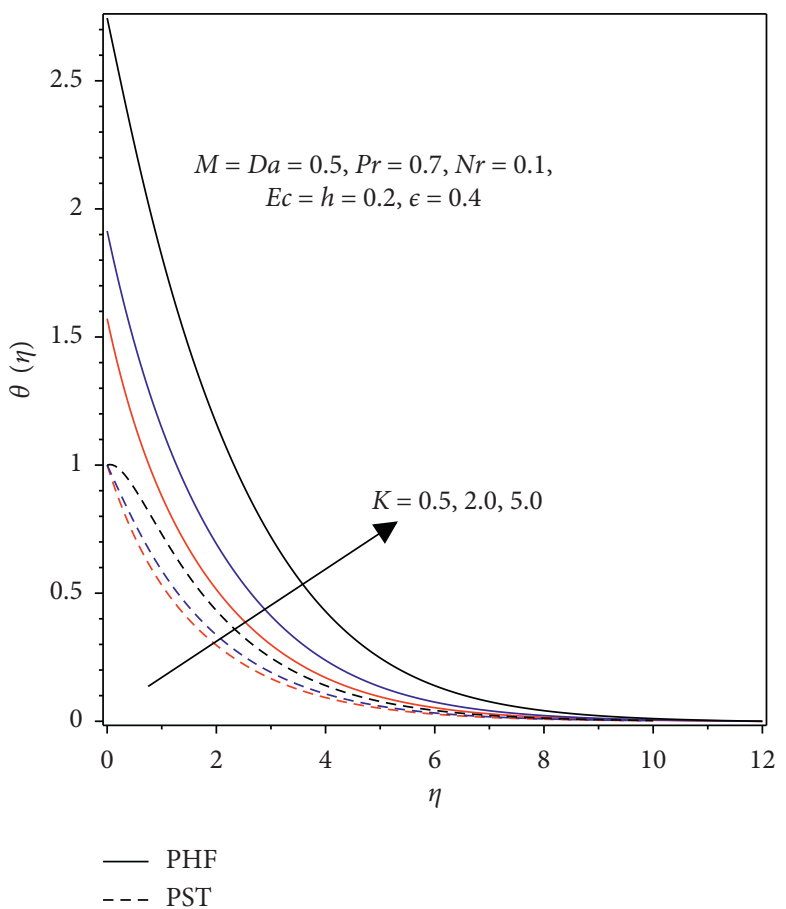

FIgURE 6: Reaction of thermal field for variation in material parameter $K$.

fluid viscosity creates resistance to the fluid motion, and consequently, heat is being generated due to the sluggishness in the flow which leads to a rise in temperature. However, the impact created by the PHF case is stronger than that of the PST case with growth in $h$. More so, it is clear that the surface temperature is lower in the absence of temperature- 


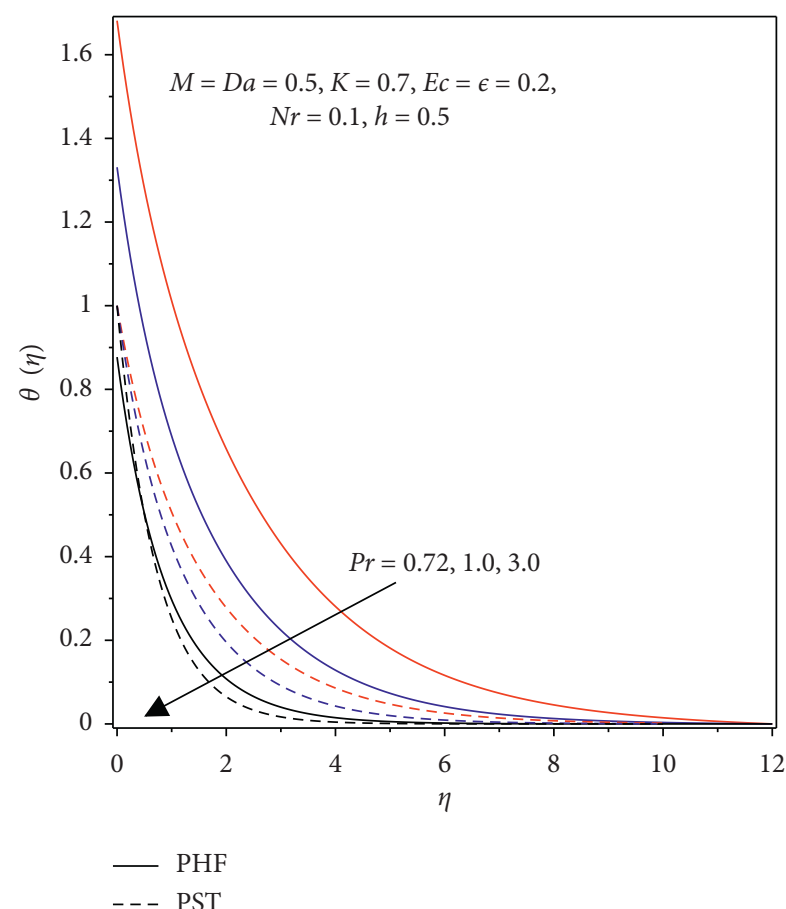

Figure 7: Impact of Prandtl number Pr on the field of temperature.

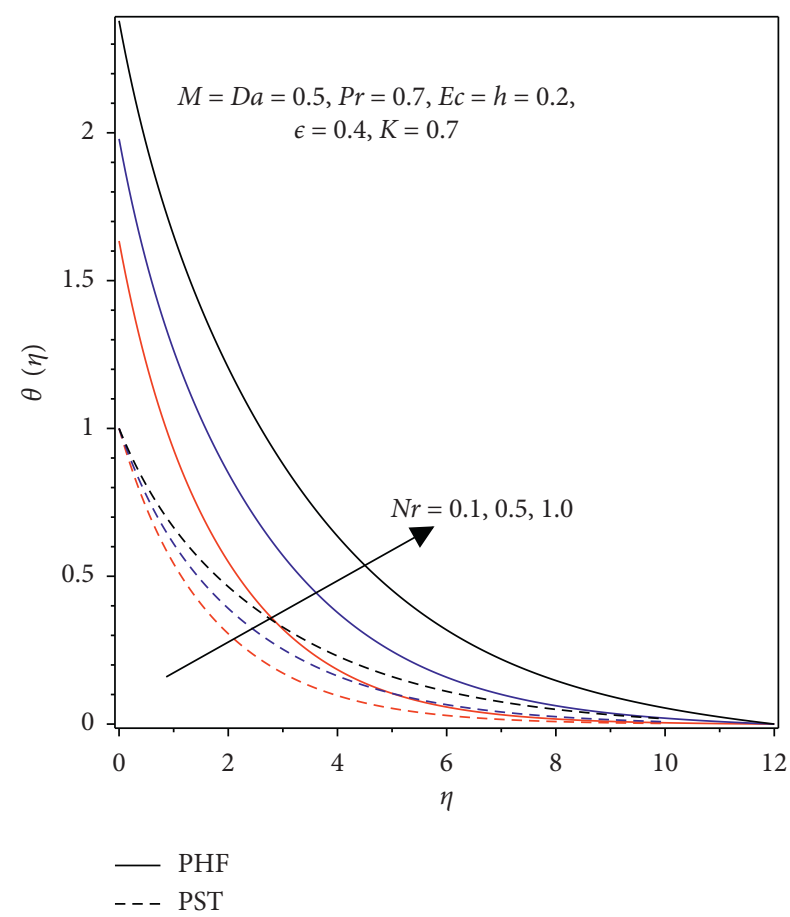

Figure 8: Influence of radiation parameter $\mathrm{Nr}$ on the profiles of temperature.

dependent viscosity parameter $h$, i.e., $h=0$, for both situations. Similarly, the plot in Figure 3 depicts that advancing the magnitude of the thermal conductivity term $\epsilon$ enables the surface temperature to appreciate which also enhances the

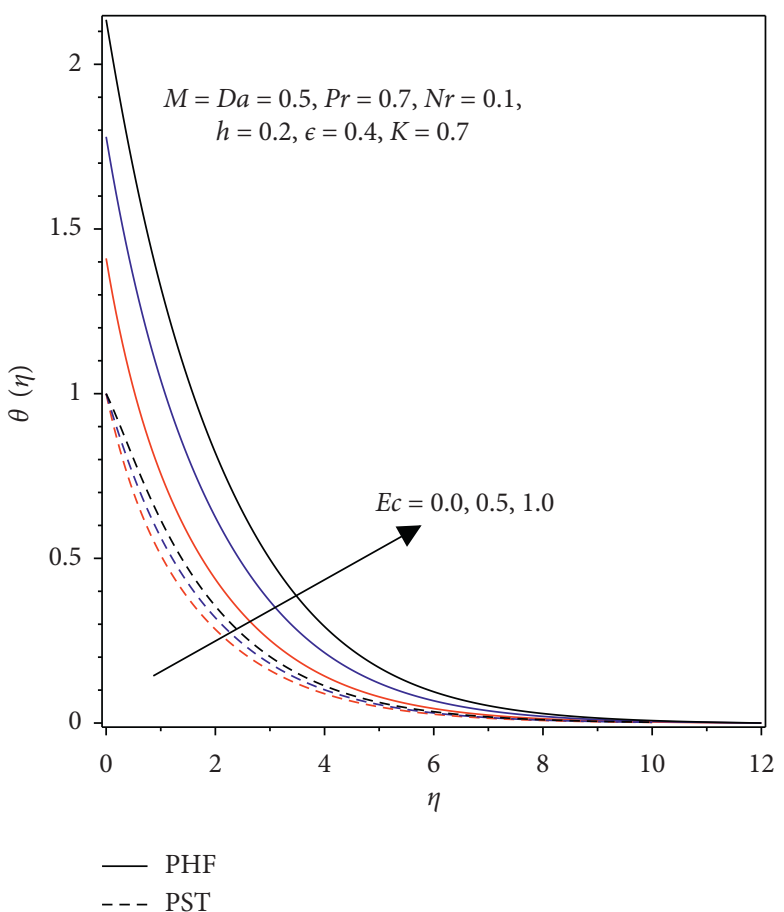

FIgURE 9: Reaction of temperature field with Eckert number $E c$.

thickness of the thermal boundary layer for both conditions. For a constant state of $\epsilon$, a lower surface temperature is witnessed as compared with the variable state. The PHF case, however, exerts a stronger influence on the temperature field than that of PST.

Figure 4 demonstrates that the surface temperature grows in both cases with rising values of the magnetic field term $M$. Physically, the application of $M$ heats up the fluid and thus lowers the heat transfer. Besides, when the magnetic field is imposed transversely on the fluid which is electrically conducting, it induces a kind of force described as Lorentz force such that the motion of the fluid is declined. In consequence of the declination to the flow imposed by the Lorentz force, the surface temperature rises as depicted in the graph.

A similar behaviour to that of $M$ is observed for varying Darcy parameter $D a$ on the temperature distribution as demonstrated in Figure 5. The graph of the dimensionless temperature against $\eta$ for variations in the material (micropolar) parameter $K$ shown in Figure 6 reveals that a growth in the magnitude of $K$ facilitates a rise in the thickness of the thermal boundary layer, and at such, there is advancement in the temperature field for the two cases examined.

Figure 7 is a sketch of the behaviour of the Prandtl number $\mathrm{Pr}$ as relates to the nondimensional temperature. Evidently, a rise in the magnitude of $P r$ produces a dampen effect on the temperature distribution and as well a decline in the thermal boundary thickness. The implication here is that a growth in $\operatorname{Pr}$ lowers the thermal boundary layer thickness which in turn diminishes the average temperature. In the physical description, the Prandtl number is inversely proportional to the thermal diffusivity, so a boost in $\mathrm{Pr}$ lowers thermal diffusion. In consequence, the surface temperature 
Table 4: Computational values of $C_{f}^{\star}, N u_{x}^{\star}$, and $C_{s}^{\star}$ for variations in $h, K$, and $P r$.

\begin{tabular}{|c|c|c|c|c|c|c|c|c|}
\hline \multicolumn{3}{|c|}{ Parameters } & \multicolumn{3}{|c|}{ PST case } & \multicolumn{3}{|c|}{ PHF case } \\
\hline$h$ & $K$ & $\operatorname{Pr}$ & $C_{f}^{\star}$ & $N u_{x}^{\star}$ & $C_{s}^{\star}$ & $C_{f}^{\star}$ & $N u_{x}^{\star}$ & $C_{s}^{\star}$ \\
\hline 0 & 1 & 1 & 1.378596 & 0.671717 & 0.620166 & 1.378596 & 0.505664 & 0.620166 \\
\hline 1.5 & 1 & 1 & 1.670709 & 0.548272 & 0.775964 & 1.788282 & 0.415882 & 0.834327 \\
\hline 4.0 & 1 & 1 & 1.800210 & 0.408643 & 0.840104 & 1.912646 & 0.381195 & 0.895340 \\
\hline 0.5 & 0.5 & 1 & 1.493594 & 0.593211 & 0.689887 & 1.627674 & 0.501681 & 0.752622 \\
\hline 0.5 & 1.5 & 1 & 1.429256 & 0.461600 & 0.639020 & 1.518529 & 0.427921 & 0.687668 \\
\hline 0.5 & 5.0 & 1 & 1.367540 & 0.001809 & 0.565978 & 1.418209 & 0.329233 & 0.595780 \\
\hline 0.5 & 1 & 1 & 1.453795 & 0.527184 & 0.662056 & 1.559297 & 0.472887 & 0.717032 \\
\hline 0.5 & 1 & 2.5 & 1.466633 & 0.846367 & 0.671385 & 1.485600 & 0.661834 & 0.681771 \\
\hline 0.5 & 1 & 4.5 & 1.475835 & 1.098439 & 0.678283 & 1.466742 & 0.873746 & 0.673121 \\
\hline
\end{tabular}

TABLE 5: Computed values of $C_{f}^{\star}, N u_{x}^{\star}$, and $C_{s}^{\star} C_{s}^{\star}$ for variations in $\epsilon, E c$, and $R$.

\begin{tabular}{|c|c|c|c|c|c|c|c|c|}
\hline \multicolumn{3}{|c|}{ Parameters } & \multicolumn{3}{|c|}{ PST case } & \multicolumn{3}{|c|}{ PHF case } \\
\hline$\epsilon$ & $E c$ & $\mathrm{Nr}$ & $C_{f}^{\star}$ & $N u_{x}^{\star}$ & $C_{s}^{\star}$ & $C_{f}^{\star}$ & $N u_{x}^{\star}$ & $C_{s}^{\star}$ \\
\hline 0 & 1 & 1 & 1.533853 & 0.741649 & 0.705545 & 1.559892 & 0.819345 & 0.719947 \\
\hline 0.5 & 1 & 1 & 1.528221 & 0.588995 & 0.701624 & 1.586188 & 0.625917 & 0.732591 \\
\hline 1.0 & 1 & 1 & 1.521419 & 0.444884 & 0.697065 & 1.620479 & 0.454651 & 0.749108 \\
\hline 0.5 & 0 & 1 & 1.458978 & 0.766506 & 0.666063 & 1.518526 & 0.635267 & 0.696908 \\
\hline 0.5 & 0.5 & 1 & 1.453795 & 0.527184 & 0.662056 & 1.559297 & 0.472889 & 0.717032 \\
\hline 0.5 & 1.0 & 1 & 1.448910 & 0.288854 & 0.658270 & 1.591234 & 0.381405 & 0.732830 \\
\hline 0.5 & 1 & 0.1 & 1.453795 & 0.527184 & 0.662056 & 1.559297 & 0.472889 & 0.717032 \\
\hline 0.5 & 1 & 1.0 & 1.449006 & 0.416175 & 0.658649 & 1.597896 & 0.361478 & 0.735897 \\
\hline 0.5 & 1 & 1.5 & 1.447372 & 0.375465 & 0.657477 & 1.615088 & 0.322920 & 0.744367 \\
\hline
\end{tabular}

declines. Increasing Prandtl parameter, therefore, enhances the rate of cooling (see Table 4) as fluids with smaller $\mathrm{Pr}$ create stronger conductivities such that heat diffuses quickly away from the heated surface. However, a sharp decrease in the surface temperature is noticed for the PHF case than that of the PST case.

It is obvious from Figure 8 that rising values of $\mathrm{Nr}$ encourages growth in the field of temperature. In line with expectation, a growth in the thermal boundary layer is encountered with rising $N r$. This reaction occurs due to a fall in the mean absorption coefficient of the Rosseland approximation as $\mathrm{Nr}$ rises. In such situation, the divergence of the radiative heat flux as well as the heat transfer into the fluid is enhanced. Besides, a rise in $N r$ has the tendency to boost the conduction influence which enables the temperature advance at every point away from the sheet. Figure 9 depicts the plot of temperature against $\eta$ for variation in the Eckert number $E c$ for both thermal situations. Advancing the magnitude of $E c$ relates to heat dissipation owing to viscous force, and at such, the heat dissipated flows towards the fluid, and in consequence, the temperature is raised. More so, the reaction is also attributed to the fact that rising values of $E c$ facilitates heat production due to the drag between the fluid particles; thereby, the internal heat generation rises and the temperature increases for both cases.

\section{Conclusion}

The current research investigates heat transfer behaviour for thermally radiating magneto-micropolar fluid passing a linearly stretching sheet embedded in a porous medium. The impact of pertinent parameters of engineering interest has been checked on the heat transfer processes with the application of PST and PHF as wall heating conditions. $\mathrm{Nu}$ merical solutions via the shooting method cum Runge-Kutta-Fehlberg integration technique have been applied for the resulting nonlinear ODEs, while the effects of the controlling parameters are analyzed and explained via graphs as well as tables. The following have been deduced from this study:

(i) As $K, \epsilon, E c$, and $R$ (or $P r$ ) increase, there is a decline (or advancement) in $C_{f}^{\star}, N u_{x}^{\star}$, and $C_{s}^{\star}$ for the case of PST. For the PHF case, however, an increase in $h, \epsilon, E c$, and $R$ (or $P r$ ) causes $C_{f}^{\star}$ and $C_{s}^{\star}$ to rise (or fall), while the transfer of heat at the surface reduces (or rises).

(ii) The transfer of heat reduces at the surface with growth in the magnitude of the material parameter $K$ for both cases, whereas a rise in $\operatorname{Pr}$ shows a reverse situation for both cases.

(iii) The prescribed heat flux (PHF) case has a more pronounced and stronger impact on the parameters across the boundary layer than that of the prescribed surface temperature (PST).

(iv) An increase in $h, \epsilon, M, R, E c$ with $D a$ thickens the thermal boundary layer thickness, whereas the opposite occurs when the magnitude $\mathrm{Pr}$ advances for both cases. 


\section{Nomenclature}

$\begin{array}{ll}u, v: \text { Velocity in } x, y \text { direction }\left(\mathrm{ms}^{-1}\right) \\ v: \quad \text { Kinematic viscosity }\left(\mathrm{m}^{2} \mathrm{~s}^{-1}\right) \\ \rho: & \text { Fluid density }\left(\mathrm{kgm}^{-3}\right) \\ \mu: & \text { Viscosity }\left(\mathrm{kgm}^{-1} \mathrm{~s}^{-1}\right) \\ \mu_{r}: & \text { Vortex viscosity } \\ \sigma: & \text { Electrical conductivity }\left(\mathrm{Sm}^{-1}\right) \\ c_{p}: & \text { Specific heat capacity }\left(\mathrm{J} / \mathrm{kgK}^{-1}\right. \\ k: & \text { Thermal conductivity }\left(\mathrm{Wm}^{-1} \mathrm{~K}^{-1}\right) \\ p: & \text { Surface boundary parameter } \\ \sigma^{\star}: & \text { Stefan-Boltzmann constant }\left(\mathrm{Wm}^{-2} \mathrm{~K}^{4}\right) \\ T: & \text { Temperature }(\mathrm{K}) \\ h_{f}: & \text { Coefficient of heat transfer } \\ T_{f}: & \text { Surface sheet temperature }(\mathrm{K}) \\ U_{w}: & \text { Velocity at the sheet }\left(\mathrm{ms}^{-1}\right) \\ U_{0}: & \text { Nonlinear stretching parameter } \\ j: & \text { Microinertia density }\left(\mathrm{kgm}^{-3}\right) \\ \gamma_{0}: & \text { Spin gradient viscosity }\left(\mathrm{m}^{2} \mathrm{~s}^{-1}\right) \\ T_{\infty}: & \text { Temperature at free stream }(\mathrm{K}) \\ B: & \text { Microrotation component }\left(\mathrm{s}^{-1}\right) \\ k^{\star}: & \text { Mean absorption coefficient }\left(\mathrm{m}^{-1}\right) .\end{array}$

\section{Data Availability}

There are no other supplementary data to supply in regard to this article.

\section{Conflicts of Interest}

The authors declare that they have no conflicts of interest.

\section{References}

[1] M. Gholinia, K. Hosseinzadeha, H. Mehrzadi, D. D. Ganji, and A. A. Ranjbar, "Investigation of MHD Eyring-Powell fluid flow over a rotating disk under effect of homogeneous-heterogeneous reactions," Case Studies in Thermal Engineering, vol. 12, pp. 1-10, 2019.

[2] A. C. Eringen, "Simple microfluids," International Journal of Engineering Science, vol. 2, no. 2, pp. 205-217, 1964.

[3] A. Eringen, "Theory of micropolar fluids," Indiana University Mathematics Journal, vol. 16, no. 1, pp. 1-18, 1966.

[4] M. Reena and U. S. Rana, "Effect of dust particles on rotating micropolar fluid heated from below saturating a porous medium," Applied Mathematics and Computation, vol. 4, pp. 189-217, 2009.

[5] G. Ahmadi, "Self-similar solution of imcompressible micropolar boundary layer flow over a semi-infinite plate," International Journal of Engineering Science, vol. 14, no. 7, pp. 639-646, 1976.

[6] T. Hayat, M. Mustafa, and S. Obaidat, "Soret and Dufour effects on the stagnation point flow of a micropolar fluid toward a stretching sheet," Journal of Fluids Engineering, vol. 133, pp. 1-9, 2011.

[7] G. Lukaszewicz, Micropolar Fluids: Theory and Applications, Birkhauser, Basel, Switzerland, 1999.

[8] J. Peddieson and R. P. McNitt, "Boundary layer theory for micropolar fluid," Recent Advances in Engineering Science, vol. 5, pp. 405-426, 1968.
[9] A. J. Wilson, "Boundary layers in micropolar liquids," Mathematical Proceedings of the Cambridge Philosophical Society, vol. 67, pp. 469-470, 1970.

[10] L. J. Crane, "Flow past a stretching plate," Zeitschrift für angewandte Mathematik und Physik ZAMP, vol. 21, no. 4, pp. 645-647, 1970.

[11] P. S. Gupta and A. S. Gupta, "Heat and mass transfer on a stretching sheet with suction or blowing," The Canadian Journal of Chemical Engineering, vol. 55, no. 6, pp. 744-746, 1977.

[12] A. Chakrabarti and A. S. Gupta, "Hydromagnetic flow and heat transfer over a stretching sheet," Quarterly of Applied Mathematics, vol. 37, no. 1, pp. 73-78, 1979.

[13] L. J. Grubka and K. M. Bobba, "Heat transfer characteristics of a continuous, stretching surface with variable temperature," Journal of Heat Transfer, vol. 107, no. 1, pp. 248-250, 1985.

[14] E. M. A. Elbashbeshy and M. A. A. Bazid, "Heat transfer in a porous medium over a stretching surface with internal heat generation and suction or injection," Applied Mathematics and Computation, vol. 158, no. 3, pp. 799-807, 2004.

[15] N. T. Eldabe, E. F. Elshehawey, E. M. E. Elbarbary, and N. S. Elgazery, "Chebyshev finite difference method for MHD flow of a micropolar fluid past a stretching sheet with heat transfer," Applied Mathematics and Computation, vol. 160, no. 2, pp. 437-450, 2005.

[16] E. Fatunmbi, A. S. Odesola, and O. Fenuga, "Heat and mass transfer of a chemically reacting MHD micropolar fluid flow over an exponentially stretching sheet with slip effects," Physical Science International Journal, vol. 18, no. 3, pp. 1-15, 2018.

[17] T. Cebeci and P. Bradshaw, Physical and Computational Aspects of Convective Heat Transfer, Springer, New York, NY, USA, 1984.

[18] A. Postelnicu, T. Groşan, and I. Pop, “The effect of variable viscosity on forced convection flow past a horizontal flat plate in a porous medium with internal heat generation," $M e$ chanics Research Communications, vol. 28, no. 3, pp. 331-337, 2001.

[19] P. M. Thakur and G. Hazarika, "Effects of variable viscosity and thermal conductivity of flow and heat transfer over a stretching surface with variable heat flux in micropolar fluid in the presence of magnetic fluid," International Journal of Science and Mathematics Research, vol. 2, pp. 554-566, 2014.

[20] S. Jana and K. Das, "Influence of variable fluid properties, thermal radiation and chemical reaction on MHD flow over a flat plate," Italian Journal of Pure and Applied Mathematics, vol. 34, pp. 20-44, 2015.

[21] T. E. Akinbobola and S. S. Okoya, "The flow of second grade fluid over a stretching sheet with variable thermal conductivity and viscosity in the presence of heat source/sink," Journal of the Nigerian Mathematical Society, vol. 34, no. 3, pp. 331-342, 2015.

[22] S. E. Ahmed and Z. Z. Rashed, "MHD natural convection in a heat generating porous medium-filled wavy enclosures using Buongiorno's nanofluid model," Case Studies in Thermal Engineerin, vol. 14, pp. 1-15, 2019.

[23] C. H. Amanulla, S. Saleem, A. Wakif, and M. M. AlQarni, "MHD Prandtl fluid flow past an isothermal permeable sphere with slip effects," Case Studies in Thermal Engineering, vol. 14, pp. 1-6, 2019.

[24] M. S. Kausar, A. Hussanan, M. Mamat, and B. Ahmad, "Boundary layer flow through Darcy-Brinkman porous medium in the presence of slip effects and porous dissipation," Symmetry, vol. 11, pp. 1-11, 2019. 
[25] A. R. Hassan, J. A. Gbadeyan, and S. O. Salawu, "The effects of thermal radiation on a reactive hydromagnetic internal heat generating fluid flow through parallel porous plates," Recent Advances in Mathematical, pp. 1-11, 2018.

[26] E. Fatunmbi and A. Adeniyan, "MHD stagnation point-flow of micropolar fluids past a permeable stretching plate in porous media with thermal radiation, chemical reaction and viscous dissipation," Journal of Advances in Mathematics and Computer Science, vol. 26, no. 1, pp. 1-19, 2018.

[27] M. R. Zangooee, K. Hosseinzadeh, and D. D. Ganji, "Hydrothermal analysis of MHD nanofluid $\left(\mathrm{TiO}_{2}-\mathrm{GO}\right)$ flow between two radiative stretchable rotating disks using AGM," Case Studies in Thermal Engineering, vol. 14, pp. 1-13, 2019.

[28] S. O. Salawu and M. S. Dada, "Radiative heat transfer of variable viscosity and thermal conductivity effects on inclined magnetic field with dissipation in a non-Darcy medium," Journal of the Nigerian Mathematical Society, vol. 35, no. 1, pp. 93-106, 2016.

[29] A. Ishak, "Thermal boundary layer flow over a stretching sheet in a micropolar fluid with radiation effect," Meccanica, vol. 45, no. 3, pp. 367-373, 2010.

[30] R. Cortell, "Combined effect of viscous dissipation and thermal radiation on fluid flows over a non-linearly stretched permeable wall," Meccanica, vol. 47, pp. 69-81, 2012.

[31] M. Gholinia, S. Gholinia, K. Hosseinzadeh, and D. D. Ganji, "Investigation on ethylene glycol Nano fluid flow over a vertical permeable circular cylinder under effect of magnetic field," Results in Physics, vol. 9, pp. 1525-1533, 2018.

[32] B. J. Gireesha, G. K. Ramesh, and C. S. Ragewadi, "Heat transfer characteristics in MHD flow of a dusty fluid over a stretching sheet with viscous dissipation," Advances in Applied Science Research, vol. 3, no. 4, pp. 2392-2401, 2012.

[33] D. Pal and M. Mondal, "Effects of temperature-dependent viscosity and variable thermal conductivity on MHD nonDarcy mixed convective diffusion of species over a stretching sheet," Journal of the Egyptian Mathematical Society, vol. 22, no. 1, pp. 123-133, 2013.

[34] F. C. Lai and F. A. Kulacki, "The effect of variable viscosity on convective heat transfer along a vertical surface in a saturated porous medium," International Journal of Heat and Mass Transfer, vol. 33, no. 5, pp. 1028-1031, 1990.

[35] M. Kumari, "Effect of variable viscosity on non-Darcy free or mixed convection flow on a horizontal surface in a saturated porous medium," International Communications in Heat and Mass Transfer, vol. 28, no. 5, pp. 723-732, 2001.

[36] K. E. Chin, R. Nazar, N. M. Arifin, and I. Pop, "Effect of variable viscosity on mixed convection boundary layer flow over a vertical surface embedded in a porous medium," International Communications in Heat and Mass Transfer, vol. 34, no. 4, pp. 464-473, 2007.

[37] T. C. Chiam, "Heat transfer in a fluid variable thermal conductivity over a linearly stretching sheet," Acta Mechanica, vol. 129, pp. 63-72, 1997.

[38] K. Das, S. Jana, and P. K. Kundu, “Thermophoretic MHD slip flow over a permeable surface with variable fluid properties," Alexandria Engineering Journal, vol. 54, no. 1, pp. 35-44, 2015.

[39] A. Adeniyan, "MHD mixed convection of a viscous dissipating and chemically reacting stagnation-point flow near a vertical permeable plate in a porous medium with thermal radiation and heat source/sink," Asian Journal of Mathematics and Applications, vol. 2015, pp. 1-23, 2015.

[40] J. Peddieson, "An application of the micropolar fluid model to the calculation of a turbulent shear flow," International Journal of Engineering Science, vol. 10, no. 1, pp. 23-32, 1972.
[41] S. K. Jena and M. N. Mathur, "Similarity solutions for laminar free convection flow of a thermomicropolar fluid past a nonisothermal vertical flat plate," International Journal of Engineering Science, vol. 19, no. 11, pp. 1431-1439, 1981.

[42] M. M. Rahman, M. J. Uddin, and A. Aziz, "Effects of variable electric conductivity and non-uniform heat source (or sink) on convective micropolar fluid flow along an inclined flat plate with surface heat flux," International Journal of Thermal Sciences, vol. 48, pp. 2331-2340, 2005.

[43] M. A. Seddeek and A. M. Salem, "Laminar mixed convection adjacent to vertical continuously stretching sheets with variable viscosity and variable thermal diffusivity," Heat and Mass Transfer, vol. 41, no. 12, pp. 1048-1055, 2005.

[44] M.-E. M. Salem, A. J. Chamkha, and M. Bayomi, "MHD flow of a micropolar fluid past a stretched permeable surface with heat generation or absorption," Nonlinear Analysis: Modelling and Control, vol. 14, no. 1, pp. 27-40, 2009.

[45] M. M. Nandeppanavar, K. Vajravelu, M. Subhas Abel, and M. N. Siddalingappa, "MHD flow and heat transfer over a stretching surface with variable thermal conductivity and partial slip," Meccanica, vol. 48, no. 6, pp. 1451-1464, 2013.

[46] H. Waqas, S. Hussain, H. Sharif, and S. Khalid, "MHD forced convective flow of micropolar fluids past a moving boundary surface with prescribed heat flux and radiation," British Journal of Mathematics \& Computer Science, vol. 21, no. 1, pp. 1-14, 2017.

[47] B. S. Attili and M. L. Syam, "Efficient shooting method for solving two point boundary value problems," Chaos, Solitons and Fractals, vol. 35, no. 5, pp. 895-903, 2008.

[48] B. Mahanthesh, B. J. Gireesha, R. S. R. Gorla, and O. D. Makinde, "Magnetohydrodynamic three-dimensional flow of nanofluids with slip and thermal radiation over a nonlinear stretching sheet: a numerical study," Neural Computing and Applications, vol. 30, no. 5, pp. 1557-1567, 2018.

[49] L. Kumar, "Finite element analysis of combined heat and mass transfer in hydromagnetic micropolar flow along a stretching sheet," Computational Materials Science, vol. 46, no. 4, pp. 841-848, 2009.

[50] R. S. Tripathy, G. C. Dash, S. R. Mishra, and M. M. Hoque, "Numerical analysis of hydromagnetic micropolar fluid along a stretching sheet embedded in porous medium with nonuniform heat source and chemical reaction," Engineering Science and Technology, an International Journal, vol. 19, no. 3, pp. 1573-1581, 2016.

[51] C.-H. Chen, "Laminar mixed convection adjacent to vertical, continuously stretching sheets," Heat and Mass Transfer, vol. 33, no. 5-6, pp. 471-476, 1998.

[52] K. Das, "Slip effects on heat and mass transfer in MHD micropolar fluid flow over an inclined plate with thermal radiation and chemical reaction," International Journal for Numerical Methods in Fluids, vol. 70, no. 1, pp. 96-113, 2012. 